\title{
ON THE SUM OF RECIPROCAL OF GENERALIZED BI-PERIODIC FIBONACCI NUMBERS
}

\author{
MUSA BAŞBÜK AND YASIN YAZLIK
}

Received 11 May, 2015

\begin{abstract}
The generalized bi-periodic Fibonacci sequence $\left\{q_{n}\right\}_{n=0}^{\infty}$ have initial conditions $q_{0}=$ $0, q_{1}=1$ and recurrence relation $q_{n}=a q_{n-1}+q_{n-2}$ (when $n$ is even and $n \geq 2$ ) or $q_{n}=$ $b q_{n-1}+q_{n-2}$ (when $n$ is odd and $n \geq 3$ ), where $a$ and $b$ are nonzero real numbers. Some well-known sequences, such as the Fibonacci sequence, the Pell sequence and the $k$-Fibonacci sequence, are special cases of this generalization. In this paper, we consider the partial infinite sum derived from the reciprocal of the generalized bi-periodic Fibonacci numbers.
\end{abstract}

2010 Mathematics Subject Classification: 11B39; 11B83

Keywords: partial infinite sum, reciprocal sums, bi-periodic Fibonacci numbers

\section{INTRODUCTION}

In recent years special sequences such as Fibonacci, Lucas, Pell and the generalizations of these sequences have attracted a great interest. Many researchers investigated new properties of these sequences $[1-3,6,9,10]$. In fact one of the most well-known omitted aforementioned sequences is the Fibonacci sequence which starts with the integer pair 0 and 1, defined by the recurrence relation $F_{n}=F_{n-1}+F_{n-2}$ for all $n \geq 2$. The Fibonacci sequence has a wide range of applications in various areas such as Mathematics, Statistics, Biology, Physics, Finance, Architecture, Computer Science, etc. Various generalizations are defined by some authors. (see [9] and the references there in for a list of references for history, properties, applications and the generalizations of the Fibonacci sequence). Edson and Yayenie [2] introduced a new generalized Fibonacci sequence that depends on two real parameters as defined below.

Definition 1. For any two nonzero real numbers $a$ and $b$, the generalized Fibonacci sequence $\left\{q_{n}\right\}_{n=0}^{\infty}$ is defined recursively by

$$
q_{n}=\left\{\begin{array}{ll}
a q_{n-1}+q_{n-2} & \text { if } n \text { is even, } \\
b q_{n-1}+q_{n-2} & \text { if } n \text { is odd, }
\end{array} \quad(n \geq 2) \quad \text { with } q_{0}=0, \quad q_{1}=1 .\right.
$$

These sequences occur in the study of continued fractions of quadratic irrationals and combinatorics on words or dynamical system theory [9]. One can get, the 
classical Fibonacci sequence when $a=b=1$, the Pell numbers when $a=b=2$ and the $k$-Fibonacci numbers $a=b=k$ for some positive integer $k$. In [2] Edson and Yayenie obtained the extended Binet's Formula for the generalized Fibonacci sequence. Later Yayenie obtained numerous new identities of the generalized Fibonacci sequences [9]. The partial infinite sum of reciprocal of these sequences has been studied by various resarchers. Ohtsuka \& Nakamura introduced the following partial infinite sum of reciprocal of Fibonacci numbers [7],

$$
\left\lfloor\left(\sum_{k=n}^{\infty} \frac{1}{F_{k}}\right)^{-1}\right\rfloor=\left\{\begin{array}{cc}
F_{n-2} & \text { if } n \text { is even and } n \geq 2, \\
F_{n-2}-1 & \text { if } n \text { is odd and } n \geq 1 .
\end{array}\right.
$$

Zhang and Wang introduced the infinite sum of reciprocal of Pell numbers [8],

$$
\left\lfloor\left(\sum_{k=n}^{\infty} \frac{1}{P_{k}}\right)^{-1}\right\rfloor=\left\{\begin{array}{cc}
P_{n-1}+P_{n-2} & \text { if } n \text { is even and } n \geq 2, \\
P_{n-1}+P_{n-2}-1 & \text { if } n \text { is odd and } n \geq 1
\end{array}\right.
$$

Holliday \& Komatsu obtained the infinite sum of reciprocal of generalized Fibonacci sequence $\left\{U_{n}(p, 1)\right\}$ [4],

$$
\left\lfloor\left(\sum_{k=n}^{\infty} \frac{1}{U_{k}}\right)^{-1}\right\rfloor=\left\{\begin{array}{cc}
U_{n}-U_{n-1} & \text { if } n \text { is even and } n \geq 2, \\
U_{n}-U_{n-1}-1 & \text { if } n \text { is odd and } n \geq 1
\end{array}\right.
$$

and Kilic \& Arikan [5] obtained similar results for more generalized higher order recursive sequences with additional one coefficient parameter.

In this article, we introduce and compute the partial infinite sum of reciprocal of generalized bi-periodic Fibonacci numbers which generalizes the infinite sums of reciprocal of the Fibonacci, generalized Fibonacci and Pell numbers obtained by Ohtsuka \& Nakamura, Holliday \& Komatsu and Zhang \& Wang respectively.

\section{MAIN RESULTS}

In this section, the partial infinite sum of reciprocal of the generalized Fibonacci numbers defined by the Definition 1 is studied. Here, $\lfloor$.$\rfloor denotes the floor function.$

\section{Theorem 1.}

$$
\text { Let } \zeta(k)=\left\{\begin{array}{l}
0 \text { if } k \text { is even, } \\
1 \text { if } k \text { is odd, }
\end{array}\right.
$$

and

$$
\psi(k)=\zeta(k+1)-\zeta(n+1)-(-1)^{n}\left\lfloor\frac{k-n}{2}\right\rfloor
$$


where $a$ and $b$ are real numbers with $a \geq b \geq 1$, then

$$
\left\lfloor\left(\sum_{k=n}^{\infty} \frac{\left(\frac{a}{b}\right)^{\psi(k)}}{q_{k}}\right)^{-1}\right\rfloor=\left\{\begin{array}{cc}
q_{n}-q_{n-1} & \text { if } n \text { is even and } n \geq 2, \\
q_{n}-q_{n-1}-1 & \text { if } n \text { is odd and } n \geq 1 .
\end{array}\right.
$$

We use the following identity given in [9] to prove Theorem 1.

Lemma 1 (Cassini's Identity).

$$
\left(\frac{a}{b}\right)^{\zeta(n+1)} q_{n-1} q_{n+1}-\left(\frac{a}{b}\right)^{\zeta(n)} q_{n}^{2}=\left(\frac{a}{b}\right)(-1)^{n},
$$

Proof of Theorem 1. Using Lemma 1, for $n \geq 1$, we have

$$
\begin{aligned}
& \frac{\left(\frac{a}{b}\right)^{\zeta(n+1)}}{q_{n}-q_{n-1}}-\frac{\left(\frac{a}{b}\right)^{\zeta(n+1)}}{q_{n}}-\frac{\left(\frac{a}{b}\right)^{\zeta(n)}}{q_{n+1}}-\frac{\left(\frac{a}{b}\right)^{\zeta(n)}}{q_{n+2}-q_{n+1}} \\
& =\frac{\left(\frac{a}{b}\right)^{\zeta(n+1)}\left(q_{n+2}-q_{n+1}\right)-\left(\frac{a}{b}\right)^{\zeta(n)}\left(q_{n}-q_{n-1}\right)}{\left(q_{n}-q_{n-1}\right)\left(q_{n+2}-q_{n+1}\right)} \\
& -\frac{\left(\frac{a}{b}\right)^{\zeta(n)} q_{n}+\left(\frac{a}{b}\right)^{\zeta(n+1)} q_{n+1}}{q_{n} q_{n+1}} \\
& =\frac{q_{n+2}\left(\left(\frac{a}{b}\right)^{\zeta(n+1)} q_{n-1} q_{n+1}-\left(\frac{a}{b}\right)^{\zeta(n)} q_{n}^{2}\right)}{q_{n} q_{n+1}\left(q_{n}-q_{n-1}\right)\left(q_{n+2}-q_{n+1}\right)} \\
& +\frac{q_{n-1}\left(\left(\frac{a}{b}\right)^{\zeta(n)} q_{n} q_{n+2}-\left(\frac{a}{b}\right)^{\zeta(n+1)} q_{n+1}^{2}\right)}{q_{n} q_{n+1}\left(q_{n}-q_{n-1}\right)\left(q_{n+2}-q_{n+1}\right)} \\
& =\frac{q_{n+2}\left(\frac{a}{b}\right)(-1)^{n}+q_{n-1}\left(\frac{a}{b}\right)(-1)^{n+1}}{q_{n} q_{n+1}\left(q_{n}-q_{n-1}\right)\left(q_{n+2}-q_{n+1}\right)} \\
& =\frac{\left(\frac{a}{b}\right)(-1)^{n}\left(q_{n+2}-q_{n-1}\right)}{q_{n} q_{n+1}\left(q_{n}-q_{n-1}\right)\left(q_{n+2}-q_{n+1}\right)} .
\end{aligned}
$$

If $n$ is even with $n \geq 2$, the right-hand side of equation (2.2) is positive. Then we get

$$
\frac{\frac{a}{b}}{q_{n}-q_{n-1}}>\frac{\frac{a}{b}}{q_{n}}+\frac{1}{q_{n+1}}+\frac{1}{q_{n+2}-q_{n+1}} .
$$

By applying inequality (2.3) repeatedly, we have

$$
\begin{aligned}
\frac{\frac{a}{b}}{q_{n}-q_{n-1}} & >\frac{\frac{a}{b}}{q_{n}}+\frac{1}{q_{n+1}}+\frac{1}{q_{n+2}-q_{n+1}} \\
& >\frac{\frac{a}{b}}{q_{n}}+\frac{1}{q_{n+1}}+\frac{1}{q_{n+2}}+\frac{\left(\frac{a}{b}\right)^{-1}}{q_{n+3}}+\frac{\left(\frac{a}{b}\right)^{-1}}{q_{n+4}-q_{n+3}}
\end{aligned}
$$




$$
>\frac{\frac{a}{b}}{q_{n}}+\frac{1}{q_{n+1}}+\frac{1}{q_{n+2}}+\frac{\left(\frac{a}{b}\right)^{-1}}{q_{n+3}}+\frac{\left(\frac{a}{b}\right)^{-1}}{q_{n+4}}+\frac{\left(\frac{a}{b}\right)^{-2}}{q_{n+5}}+\frac{\left(\frac{a}{b}\right)^{-2}}{q_{n+6}}+\cdots .
$$

Thus, we obtain

$$
\sum_{k=n}^{\infty} \frac{\left(\frac{a}{b}\right)^{\psi(k)}}{q_{k}}<\frac{1}{q_{n}-q_{n-1}} .
$$

Similarly, if $n$ is odd with $n \geq 1$, then

$$
\frac{1}{q_{n}-q_{n-1}}<\frac{1}{q_{n}}+\frac{\frac{a}{b}}{q_{n+1}}+\frac{\frac{a}{b}}{q_{n+2}-q_{n+1}} .
$$

By applying inequality (2.5) repeatedly, we have

$$
\begin{aligned}
\frac{1}{q_{n}-q_{n-1}} & <\frac{1}{q_{n}}+\frac{\frac{a}{b}}{q_{n+1}}+\frac{\frac{a}{b}}{q_{n+2}-q_{n+1}} \\
& <\frac{1}{q_{n}}+\frac{\frac{a}{b}}{q_{n+1}}+\frac{\frac{a}{b}}{q_{n+2}}+\frac{\left(\frac{a}{b}\right)^{2}}{q_{n+3}}+\frac{\left(\frac{a}{b}\right)^{2}}{q_{n+4}-q_{n+3}} \\
& <\frac{1}{q_{n}}+\frac{\frac{a}{b}}{q_{n+1}}+\frac{\frac{a}{b}}{q_{n+2}}+\frac{\left(\frac{a}{b}\right)^{2}}{q_{n+3}}+\frac{\left(\frac{a}{b}\right)^{2}}{q_{n+4}}+\frac{\left(\frac{a}{b}\right)^{3}}{q_{n+5}}+\frac{\left(\frac{a}{b}\right)^{3}}{q_{n+6}}+\cdots .
\end{aligned}
$$

Hence, we obtain

$$
\sum_{k=n}^{\infty} \frac{\left(\frac{a}{b}\right)^{\psi(k)}}{q_{k}}>\frac{1}{q_{n}-q_{n-1}} .
$$

On the other hand, if $n$ is even with $n \geq 2$, by using Lemma 1, then we have

$$
\begin{aligned}
& \frac{\left(\frac{a}{b}\right)^{\zeta(n+1)}-\frac{\left(\frac{a}{b}\right)^{\zeta(n+1)}}{q_{n}}-\frac{\left(\frac{a}{b}\right)^{\zeta(n)}}{q_{n+1}}-\frac{\left(\frac{a}{b}\right)^{\zeta(n)}}{q_{n+2}-q_{n+1}+1}}{=\frac{\frac{a}{b}\left(q_{n+2}-q_{n+1}+1\right)-\left(q_{n}-q_{n-1}+1\right)}{\left(q_{n}-q_{n-1}+1\right)\left(q_{n+2}-q_{n+1}+1\right)}} \\
& -\frac{q_{n}+\frac{a}{b} q_{n+1}}{q_{n} q_{n+1}} \\
& =\frac{q_{n+2}\left(\frac{a}{b} q_{n-1} q_{n+1}-q_{n}^{2}\right)}{q_{n} q_{n+1}\left(q_{n}-q_{n-1}+1\right)\left(q_{n+2}-q_{n+1}+1\right)} \\
& +\frac{q_{n-1}\left(q_{n} q_{n+2}-\frac{a}{b} q_{n+1}^{2}\right)}{q_{n} q_{n+1}\left(q_{n}-q_{n-1}+1\right)\left(q_{n+2}-q_{n+1}+1\right)} \\
& +\frac{\left(\frac{a}{b} q_{n-1} q_{n+1}-q_{n}^{2}\right)}{q_{n} q_{n+1}\left(q_{n}-q_{n-1}+1\right)\left(q_{n+2}-q_{n+1}+1\right)} \\
& -\frac{\left(q_{n} q_{n+2}-\frac{a}{b} q_{n+1}^{2}\right)}{q_{n} q_{n+1}\left(q_{n}-q_{n-1}+1\right)\left(q_{n+2}-q_{n+1}+1\right)}
\end{aligned}
$$




$$
\begin{aligned}
& -\frac{q_{n}+\frac{a}{b} q_{n+1}+\left(\frac{a}{b} q_{n+1} q_{n+2}-q_{n-1} q_{n}\right)}{q_{n} q_{n+1}\left(q_{n}-q_{n-1}+1\right)\left(q_{n+2}-q_{n+1}+1\right)} \\
& =-\frac{-\frac{a}{b} q_{n+2}+\frac{a}{b} q_{n-1}-2 \frac{a}{b}+\left(q_{n}+\frac{a}{b} q_{n+1}\right)+\left(\frac{a}{b} q_{n+1} q_{n+2}-q_{n-1} q_{n}\right)}{q_{n} q_{n+1}\left(q_{n}-q_{n-1}+1\right)\left(q_{n+2}-q_{n+1}+1\right)}<0 .
\end{aligned}
$$

Hence, we obtain,

$$
\frac{\frac{a}{b}}{q_{n}-q_{n-1}+1}<\frac{\frac{a}{b}}{q_{n}}+\frac{1}{q_{n+1}}+\frac{1}{q_{n+2}-q_{n+1}+1}
$$

By applying inequality (2.7) repeatedly as follows

$$
\begin{aligned}
\frac{\frac{a}{b}}{q_{n}-q_{n-1}+1} & <\frac{\frac{a}{b}}{q_{n}}+\frac{1}{q_{n+1}}+\frac{1}{q_{n+2}-q_{n+1}+1} \\
& <\frac{\frac{a}{b}}{q_{n}}+\frac{1}{q_{n+1}}+\frac{1}{q_{n+2}}+\frac{\left(\frac{a}{b}\right)^{-1}}{q_{n+3}}+\frac{\left(\frac{a}{b}\right)^{-1}}{q_{n+4}-q_{n+3}+1} \\
& <\frac{\frac{a}{b}}{q_{n}}+\frac{1}{q_{n+1}}+\frac{1}{q_{n+2}}+\frac{\left(\frac{a}{b}\right)^{-1}}{q_{n+3}}+\frac{\left(\frac{a}{b}\right)^{-1}}{q_{n+4}}+\frac{\left(\frac{a}{b}\right)^{-2}}{q_{n+5}}+\frac{\left(\frac{a}{b}\right)^{-2}}{q_{n+6}}+\cdots,
\end{aligned}
$$

we get

$$
\frac{1}{q_{n}-q_{n-1}+1}<\sum_{k=n}^{\infty} \frac{\left(\frac{a}{b}\right)^{\psi(k)}}{q_{k}} .
$$

Thus, we obtain

$$
\frac{1}{q_{n}-q_{n-1}+1}<\sum_{k=n}^{\infty} \frac{\left(\frac{a}{b}\right)^{\psi(k)}}{q_{k}}<\frac{1}{q_{n}-q_{n-1}}
$$

and

$$
q_{n}-q_{n-1}<\left(\sum_{k=n}^{\infty} \frac{\left(\frac{a}{b}\right)^{\psi(k)}}{q_{k}}\right)^{-1}<q_{n}-q_{n-1}+1
$$

Therefore

$$
\left\lfloor\left(\sum_{k=n}^{\infty} \frac{\left(\frac{a}{b}\right)^{\psi(k)}}{q_{k}}\right)^{-1}\right\rfloor=q_{n}-q_{n-1} .
$$

The odd case can be considered in a similar way, by using Lemma 1 as follows

$$
\begin{aligned}
& \frac{\left(\frac{a}{b}\right)^{\zeta(n+1)}}{q_{n}-q_{n-1}-1}-\frac{\left(\frac{a}{b}\right)^{\zeta(n+1)}}{q_{n}}-\frac{\left(\frac{a}{b}\right)^{\zeta(n)}}{q_{n+1}}-\frac{\left(\frac{a}{b}\right)^{\zeta(n)}}{q_{n+2}-q_{n+1}-1} \\
& =\frac{\frac{a}{b} q_{n-1}-\frac{a}{b} q_{n+2}+\frac{2 a}{b}-\left(\frac{a}{b} q_{n}+q_{n+1}\right)+\left(q_{n+1} q_{n+2}-\frac{a}{b} q_{n-1} q_{n}\right)}{q_{n} q_{n+1}\left(q_{n}-q_{n-1}-1\right)\left(q_{n+2}-q_{n+1}-1\right)}>0 .
\end{aligned}
$$


Then we have,

$$
\frac{1}{q_{n}-q_{n-1}-1}>\frac{1}{q_{n}}+\frac{\frac{a}{b}}{q_{n+1}}+\frac{\frac{a}{b}}{q_{n+2}-q_{n+1}-1} .
$$

By applying inequality (2.12) repeatedly

$$
\begin{aligned}
\frac{1}{q_{n}-q_{n-1}-1} & >\frac{1}{q_{n}}+\frac{\frac{a}{b}}{q_{n+1}}+\frac{\frac{a}{b}}{q_{n+2}-q_{n+1}-1} \\
& >\frac{1}{q_{n}}+\frac{\frac{a}{b}}{q_{n+1}}+\frac{\frac{a}{b}}{q_{n+2}}+\frac{\left(\frac{a}{b}\right)^{2}}{q_{n+3}}+\frac{\left(\frac{a}{b}\right)^{2}}{q_{n+4}-q_{n+3}-1} \\
& >\frac{1}{q_{n}}+\frac{\frac{a}{b}}{q_{n+1}}+\frac{\frac{a}{b}}{q_{n+2}}+\frac{\left(\frac{a}{b}\right)^{2}}{q_{n+3}}+\frac{\left(\frac{a}{b}\right)^{2}}{q_{n+4}}+\frac{\left(\frac{a}{b}\right)^{3}}{q_{n+5}}+\frac{\left(\frac{a}{b}\right)^{3}}{q_{n+6}}+\cdots
\end{aligned}
$$

we have

$$
\frac{1}{q_{n}-q_{n-1}-1}>\sum_{k=n}^{\infty} \frac{\left(\frac{a}{b}\right)^{\psi(k)}}{q_{k}}
$$

Thus, we obtain

$$
\frac{1}{q_{n}-q_{n-1}-1}>\sum_{k=n}^{\infty} \frac{\left(\frac{a}{b}\right)^{\psi(k)}}{q_{k}}>\frac{1}{q_{n}-q_{n-1}}
$$

and

$$
q_{n}-q_{n-1}-1<\left(\sum_{k=n}^{\infty} \frac{\left(\frac{a}{b}\right)^{\psi(k)}}{q_{k}}\right)^{-1}<q_{n}-q_{n-1}
$$

Therefore

$$
\left\lfloor\left(\sum_{k=n}^{\infty} \frac{\left(\frac{a}{b}\right)^{\psi(k)}}{q_{k}}\right)^{-1}\right\rfloor=q_{n}-q_{n-1}-1,
$$

which completes the proof.

\section{OPEN PROBLEM}

For any integer $m \geq 2, a$ and $b$ are real numbers with $a \geq b \geq 1$, whether there exists a computational formula for

$$
\left[\left(\sum_{k=n}^{\infty} \frac{\left(\frac{a}{b}\right)^{\psi(k)}}{q_{k}^{m}}\right)^{-1}\right\rfloor
$$

where

$$
\zeta(k)= \begin{cases}0 & \text { if } k \text { is even } \\ 1 & \text { if } k \text { is odd }\end{cases}
$$


and

$$
\psi(k)=\zeta(k+1)-\zeta(n+1)-(-1)^{n}\left\lfloor\frac{k-n}{2}\right\rfloor,
$$

is an open problem, we suggest the interested readers to study this problem it with us.

\section{REFERENCES}

[1] G. Bilgici, "Two generalizations of Lucas sequence," Applied Mathematics and Computation, vol. 245, pp. 526-538, 2014, doi: 10.1016/j.amc.2014.07.111.

[2] M. Edson and O. Yayenie, "A new generalization of Fibonacci sequences and extended Binet's formula," Integers, vol. 9, pp. 639-654, 2009, doi: 10.1515/INTEG.2009.051.

[3] S. Falcon and A. Plaza, "On the Fibonacci k-numbers of arithmetic indexes," Applied Mathematics and Computation, vol. 208, pp. 180-185, 2009, doi: 10.1016/j.amc.2008.11.031.

[4] S. Holliday and T. Komatsu, "On the sum of reciprocal generalized Fibonacci numbers," Integers, vol. 11, no. 4, pp. 441-455, 2011.

[5] E. Kilic and T. Arikan, "More on the infinite sum of reciprocal usual Fibonacci, Pell and higher order recurrences," Applied Mathematics and Computation, vol. 219, pp. 7783-7788, 2013, doi: 10.1016/j.amc.2013.02.003.

[6] T. Mansour, "A formula for the generating functions of powers of Horadam's sequence," Australasian Journal of Combinatorics, vol. 30, pp. 207-212, 2004.

[7] H. Ohtsuka and S. Nakamura, "On the sum of reciprocal Fibonacci numbers," Fibonacci Quart., vol. 46/47, pp. 153-159, 2008/2009.

[8] Z. Wenpeng and W. Tingting, "The infinite sum of reciprocal Pell numbers," Applied Mathematics and Computation, vol. 218, no. 10, pp. 6164-6167, 2012.

[9] O. Yayenie, "A note on generalized Fibonacci sequences," Applied Mathematics and Computation, vol. 217, pp. 5603-5611, 2011, doi: 10.1016/j.amc.2010.12.038.

[10] Y. Yazlik and N. Taskara, "A note on generalized k-Horadam sequence," Computers and Mathematics with Applications, vol. 63, pp. 36-41, 2012, doi: 10.1016/j.camwa.2011.10.055.

Authors' addresses

Musa Başbük

Nevşehir Hacı Bektaş Veli University, Department of Mathematics, Zubeyde Hanım St., 50300 Nevşehir, Turkey

E-mail address: mbasbuk@gmail.com

Yasin Yazlık

Nevşehir Hacı Bektaş Veli University, Department of Mathematics, Zubeyde Hanım St., 50300 Nevşehir, Turkey

E-mail address: yyazlikenevsehir.edu.tr 